\title{
Neurochemical phenotype of cytoglobin-expressing neurons in the rat hippocampus
}

\author{
CHRISTIAN ANSGAR HUNDAHL ${ }^{1,2}$, JAN FAHRENKRUG $^{3}$ and JENS HANNIBAL $^{3}$ \\ ${ }^{1}$ Department of Physiology, Institute of Bio- and Translational Medicine; ${ }^{2}$ Centre for Excellence in Translation Medicine, \\ University of Tartu, Tartu 50411, Estonia; ${ }^{3}$ Department of Clinical Biochemistry, Bispebjerg Hospital, \\ Faculty of Health and Medical Sciences, University of Copenhagen, Copenhagen 2400, Denmark
}

Received April 20, 2014; Accepted June 3, 2014

DOI: $10.3892 /$ br.2014.299

\begin{abstract}
Cytoglobin (Cygb), a novel oxygen-binding protein, is expressed in the majority of tissues and has been proposed to function in nitric oxide (NO) metabolism in the vasculature and to have cytoprotective properties. However, the overall functions of $\mathrm{Cygb}$ remain elusive. Cygb is also expressed in a subpopulation of brain neurons. Recently, it has been shown that stress upregulates Cygb expression in the brain and the majority of neuronal nitric oxide synthase (nNOS)-positive neurons, an enzyme that produces NO, co-express Cygb. However, there are more neurons expressing Cygb than nNOS, thus a large number of Cygb neurons remain uncharacterized by the neurochemical content. The aim of the present study was to provide an additional and more detailed neurochemical phenotype of Cygb-expressing neurons in the rat hippocampus. The rat hippocampus was chosen due to the abundance of Cygb, as well as this limbic structure being an important target in a number of neurodegenerative diseases. Using triple immunohistochemistry, it was demonstrated that nearly all the parvalbumin- and heme oxygenase 1-positive neurons co-express Cygb and to a large extent, these neuron populations are distinct from the population of Cygb neurons co-expressing nNOS. Furthermore, it was shown that the majority of neurons expressing somastostatin and vasoactive intestinal peptide also co-express Cygb and nNOS. Detailed information regarding the neurochemical phenotype of Cygb neurons in the hippocampus can be a valuable tool in determining the function of Cygb in the brain.
\end{abstract}

Correspondence to: Dr Christian Ansgar Hundahl, Department of Physiology, Institute of Bio- and Translational Medicine, University of Tartu, 19 Ravila Street, Tartu 50411, Estonia

E-mail: c.hundahl@gmail.com

Key words: cytoglobin, hippocampus, immunohistochemistry, neurochemistry

\section{Introduction}

The hippocampus is of paramount importance as it controls a wide range of physiological processes, including learning and memory, spatial navigation and neurological diseases, such as Alzheimers's disease (AD), depression, schizophrenia and stroke, which often target the hippocampus and have profound effects on physiology (1-4). The function of the hippocampus has been extensively studied from a behavioral, biochemical and neuroanatomical perspective, establishing the hippocampus as an excellent model for studying the function of a protein. Cytoglobin (Cygb) was identified as the fourth vertebrate heme-globin in $2002(5,6)$. Cygb has, despite low sequence homology with the canonical hemoglobin and myoglobin, retained the classical globin fold and can reversibly bind oxygen and other diatomic gasses (7). Cygb is expressed in a number of tissues and is also found in neurons of the brain where Cygb is localized in the soma, neuronal processes and nuclei $(8,9)$. Within the mouse brain, Cygb is expressed in distinct areas with large regional differences in the expression levels. The areas with pronounced Cygb expression are the hippocampus, reticular thalamic nucleus (RT), habenula, laterodorsal tegmental nucleus and the pedunculopontine tegmental nucleus $(8,10,11)$. The Cygb expression patterns in the mouse brain were recently determined to be identical in the rat and human brain, demonstrating that the rodent brain can be used as a translational model for studying Cygb in humans, at least at the anatomical level $(12,13)$. The function of Cygb remains largely unknown, but several studies have linked Cygb to reactive oxygen/nitrogen species (RNS) nitric oxide (NO) scavenging (14-20). Furthermore, Cygb overexpression protects against ischemic cell death in vivo (21), although not when expressed at endogenous levels (22). In our previous studies, Cygb-immunoreactivity (ir) was shown to be highly co-localized with one of the enzymes producing NO, namely neuronal nitric oxide synthase (nNOS), in the mouse brain (9) and found that the majority of the nNOS-ir neurons of the rat hippocampus co-localized with Cygb-ir (13). However, due to the larger number of Cygb-ir cells in the hippocampus, the majority of Cygb-ir cells remain uncharacterized (13).

The aim of the present study was to extend our previous study (13) in the rat hippocampus by providing a detailed neurochemical phenotype of the Cygb-ir neurons in 
association with the subpopulation co-expressing nNOS Knowledge regarding the neurochemical phenotypes of neurons expressing the protein of interest can be a valuable tool, as it will allow the investigator to determine if the protein of interest is primarily co-localized with one other protein or groups of proteins associated with known specific functions or pathways. This information can then be used to test functional hypotheses regarding the protein of interest by investigating whether affecting the functions/pathways of the co-expressing proteins will also affect the protein of interest.

\section{Materials and methods}

Animals. Six male Wistar rats (250 g) from Taconic (Denmark), were used in the experiment. All the rats were perfusion-fixed in $4 \%$ paraformaldehyde and the brains were dissected and post-fixed in the same fixative for $24 \mathrm{~h}$ at $4^{\circ} \mathrm{C}$. The brains were cryo-protected with $30 \%$ sucrose in phosphate-buffered saline for five days and stored at $-80^{\circ} \mathrm{C}$ until required. The brains were cryo-sectioned in $40-\mu \mathrm{m}$ coronal sections in a series of five sections. Animal care and all the experimental procedures were conducted in accordance to the principles of Laboratory Animal Care (Law on Animal Experiments in Denmark, publication 1306, November 23, 2007) and approved by the Faculty of Health, University of Copenhagen (Copenhagen, Denmark).

Immunohistochemistry (IHC). The IHC protocol has been described previously (23). The primary antibodies employed for IHC were: i) Rabbit anti-Cygb [in-house, code\# 5092/6, 1:3,000 dilution and characterized previously $(9,12,13)]$; ii) sheep anti-nNOS [Dr Emson, University of Cambridge (Cambridge, UK), 1:3,000 dilution and characterized previously $(24,25)]$. nNOS produces the gas-neurotransmitter NO, which is involved in a number of physiological and pathological processes, including vasodilatation and RNS-mediated damage. iii) Goat anti-somastostatin (SOMA) [code\# sc-7819, 1:1,000 dilution and characterized previously (26,27); Santa Cruz Biotechnology, Inc., Santa Cruz, CA, USA]. SOMA is a neuroendocrine peptide hormone and regulates a number of secondary hormones via its $\mathrm{G}$ protein-coupled receptors. iv) Rabbit anti-vasoactive intestinal peptide (VIP) [in-house, code\# 291E-3, 1:15,000 dilution and characterized previously (28)]. VIP is a major regulatory peptide in the brain and is involved in a number of processes, including circadian and neuroendocrine control. v) Rabbit anti-parvalbumin (PV) [code\# PV25, 1:40,000 dilution and characterized previously (29,30); Swant, Swizerland]. PV is a calcium-binding protein involved in numerous physiological processes. vi) Rabbit anti-heme oxygenase 1 (HO-1) [code\# ADI-SPA-895, 1:60,000 dilution and characterized previously (10,31); Enzo Life Sciences, AH Diagnostics AS, Aarhus, Denmark]. HO-1 is an enzyme that catalyzes the degradation of heme groups to produce biliverdin, iron and the gas-neurotransmitter carbon monoxide. The primary antibodies were detected with either a donkey anti-sheep Alexa-488 or 568 (code\# A11015 and A21009, 1:800 dilution; Life Technologies, Carlsbad, CA, USA), donkey anti-rabbit Alexa-594 or 647 (code\# A21207 and A31573, 1:800 dilution; Life Technologies) and donkey anti-goat Alexa-649 (code\# 705-606-147, 1:300 dilution;
Jackson Immunoresearch Laboratories, Inc., West Grove, PA, USA). When two rabbit primary antibodies were used in continuation, a previously described protocol (23) was used. Briefly, following the initial block of endogen peroxide with $\mathrm{H}_{2} \mathrm{O}_{2}$ treatment, the first primary antibody, which was highly diluted, was detected with a biotin-conjugated donkey anti-rabbit (code\# 711-066-152, 1:800 dilution; Jackson Immunoresearch Laboratories, Inc.) followed by the avidin-biotin complex (code\# PK-6100; Vector Laboratories, Peterborough, UK), the Tyramide Signal Amplification system (code\# NEL700001KT; Perkin-Elmer, Waltham, MA, USA) and visualized with a strepavidin-conjugated $\mathrm{Cy} 2$ or $\mathrm{Cy} 5$ antibody (code\# 016-220-084 and 016-170-085, 1:800 dilution; Jackson Immunoresearch Laboratories, Inc.). The second primary antibody was detected with a donkey anti-rabbit Alexa-594.

Image analyses. Images were captured using an iMIC confocal microscope (FEI Munich GmbH, Germany) equipped with the appropriate filter settings for detecting 4',6-diamidino-2-phenylindole and CY2/Alexa-488, and CY3/Alexa-594 and CY5/Alexa-640. For the overview images captured in the $\mathrm{x} 10$ wide-field section of the microscope and 9x3 images were stitched together using the LA-stitch module of Fiji (ImageJ 1.47 64-bit, National Institutes of Health, Bethesda, MD, USA). The image analysis in the higher magnification, the images were captured by the spinning disk confocal section of the microscope. The Z-stacks of $\sim 60-70$ images were separated in the Z-level by $0.5 \mu \mathrm{m}$ and deconvoluted in AutoQuant X (version 3.02; Media Cybernetics, Inc., Rockville, MD, USA) and the localization of dendritic processes and cell bodies were further analyzed using the co-localization module of IMARIS (version 7.6.4; Bitplane USA, South Windsor, CT, USA). Finally, the images were corrected for brightness and contrast in Adobe Photoshop CS5 (Adobe Systems, Mountain View, CA, USA), and extended and mounted into plates using Adobe Illustrator CS5 (Adobe Systems).

\section{Results}

Cygb localization and co-localization. Intense Cygb-ir was observed throughout the rostral-caudal extend and in the majority of the hippocampal structures, with the highest density in the dentate gyrus (DG) and hilus (Fig. 1A). In the pyramidal cell layer (Py), in fields CA1-CA3, evident Cygb-ir was detected in the cell soma, nuclei and processes of the pyramidal cells (Fig. 1A). Intense nNOS-ir neurons were observed in the DG and CA1-CA3 (Fig. 1B). PV-ir interneurons and processes were observed in the same areas as Cygb-ir. The vast majority co-stored Cygb-ir, and a subset also co-stored nNOS-ir (Fig. 1B and D). The majority of the stratum radiatum ( $\mathrm{Rad}$ ) nNOS-ir cells did not contain Cygb-ir (Fig. 1D). Intense HO-1-ir cells were observed in the DG, as well as in a few cells of the CA1-CA3 (Fig. 2B), where almost all the cells co-stored Cygb-ir and a small subset also co-stored nNOS-ir (Fig. 2D). A few medium-intensity stained VIP-ir cells were observed to be scattered in DG and CA1-CA3 (Fig. 3B), where the majority of cells co-expressed Cygb-ir and nNOS-ir (Fig. 3D). SOMA-ir was highly expressed throughout the hippocampus (Fig. 4B) and was found in the 

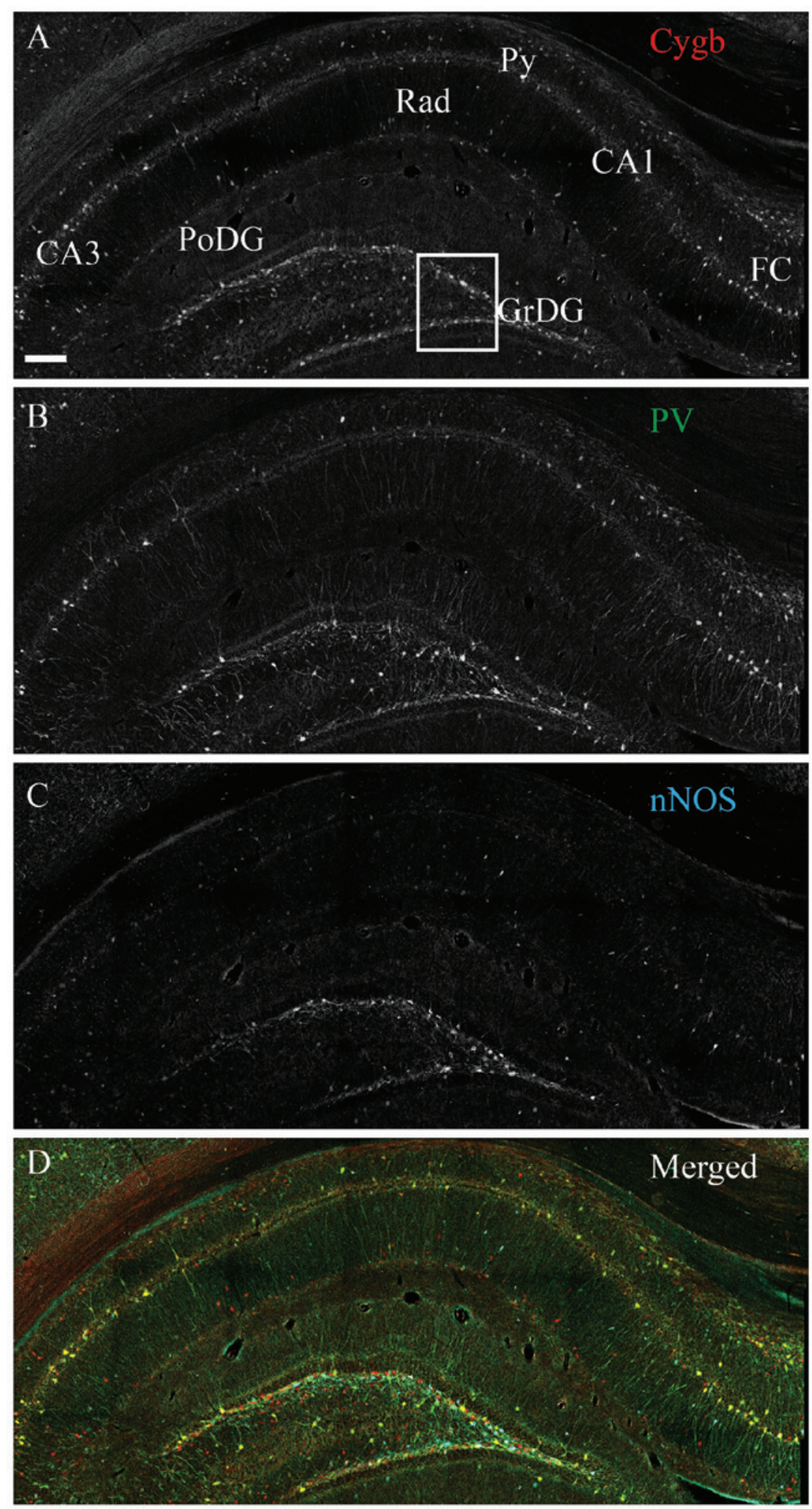

Figure 1. Cytoglobin (Cygb), parvalbumin (PV) and neuronal nitric oxide synthase (nNOS) expression in the rat hippocampus. Immunohistochemical staining of (A) Cygb, (B) PV and (C) nNOS. (D) The merged image of A-C is shown. A high degree of co-localization was observed between Cygb-immunoreactivit y (ir) (red) and PV-ir (green) throughout the hippocampus and the majority of the PV-ir neurons did not co-localize with nNOS-ir (cyan). The area within the white box is magnified in Fig. 6. CA1-3, corun ammonis 1-3; FC, fasio larumcinereum; GrDG, striatum granulosum of dentate gyrus; PoDG, polymorph layer of DG; Py, pyramidal cell layer; Rad, stratum radiatum. (A-D) Scale bar, $200 \mu \mathrm{m}$.

majority of the cells that also expressed Cygb-ir and nNOS-ir, although a subset only co-stored Cygb-ir (Fig. 4D). Outside the hippocampus, such as the RT, a high degree of co-localization between Cygb-ir and the neurochemical marker, PV, was also observed (Fig. 5).
Image analysis. The Z-stack image analysis of the areas within the white squares in Figs. 1-4 confirmed the co-localization between Cygb-ir and the neurochemical markers, and confirmed that the majority of the cells co-expressing Cygb-ir/PV-ir and Cygb-ir/HO-1-ir did not express nNOS-ir 

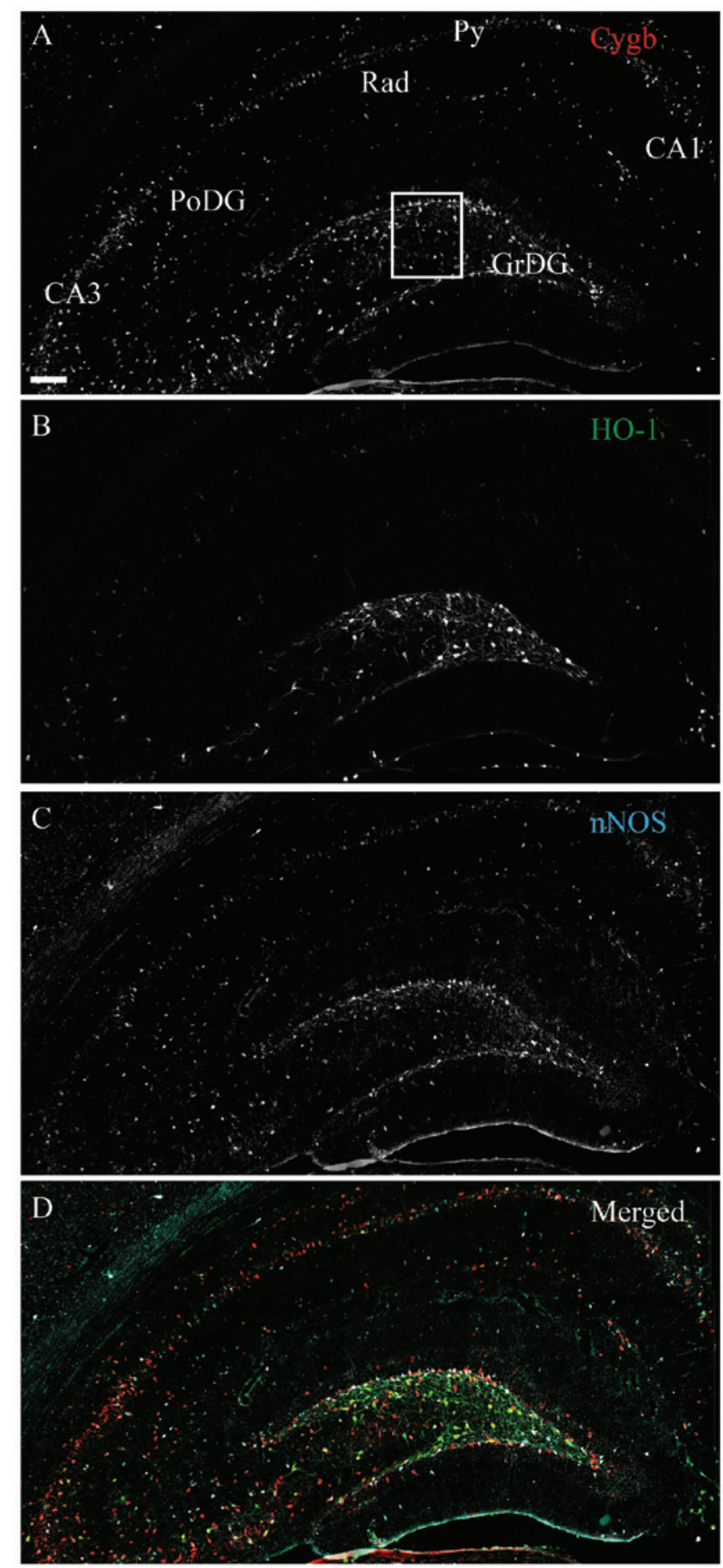

Figure 2. Cytoglobin (Cygb), heme-oxygenase-1 (HO-1) and neuronal nitric oxide synthase (nNOS) expression in the rat hippocampus. Immunohistochemical staining of (A) Cygb, (B) HO-1 and (C) nNOS (D) The merged image of A-C is shown. A high degree of co-localization is shown between Cygb-immunoreactivity (ir) (red) and HO-1-ir (green) in the DG. The majority of nNOS-ir (cyan) neurons did not co-localize with HO-1-ir. The area within the white box is magnified in Fig. 6. CA1-3, corun ammonis 1-3; GrDG, striatum granulosum of dentate gyrus; PoDG, polymorph layer of DG; Py, pyramidal cell layer; Rad, stratum radiatum. (A-D) Scale bar, $200 \mu \mathrm{m}$.

(Fig. 6A-H). Similarly, the high degree of co-localization between VIP-ir, SOMA-ir and Cygb-ir was also confirmed (Fig. 6I-L and M-P, respectively).
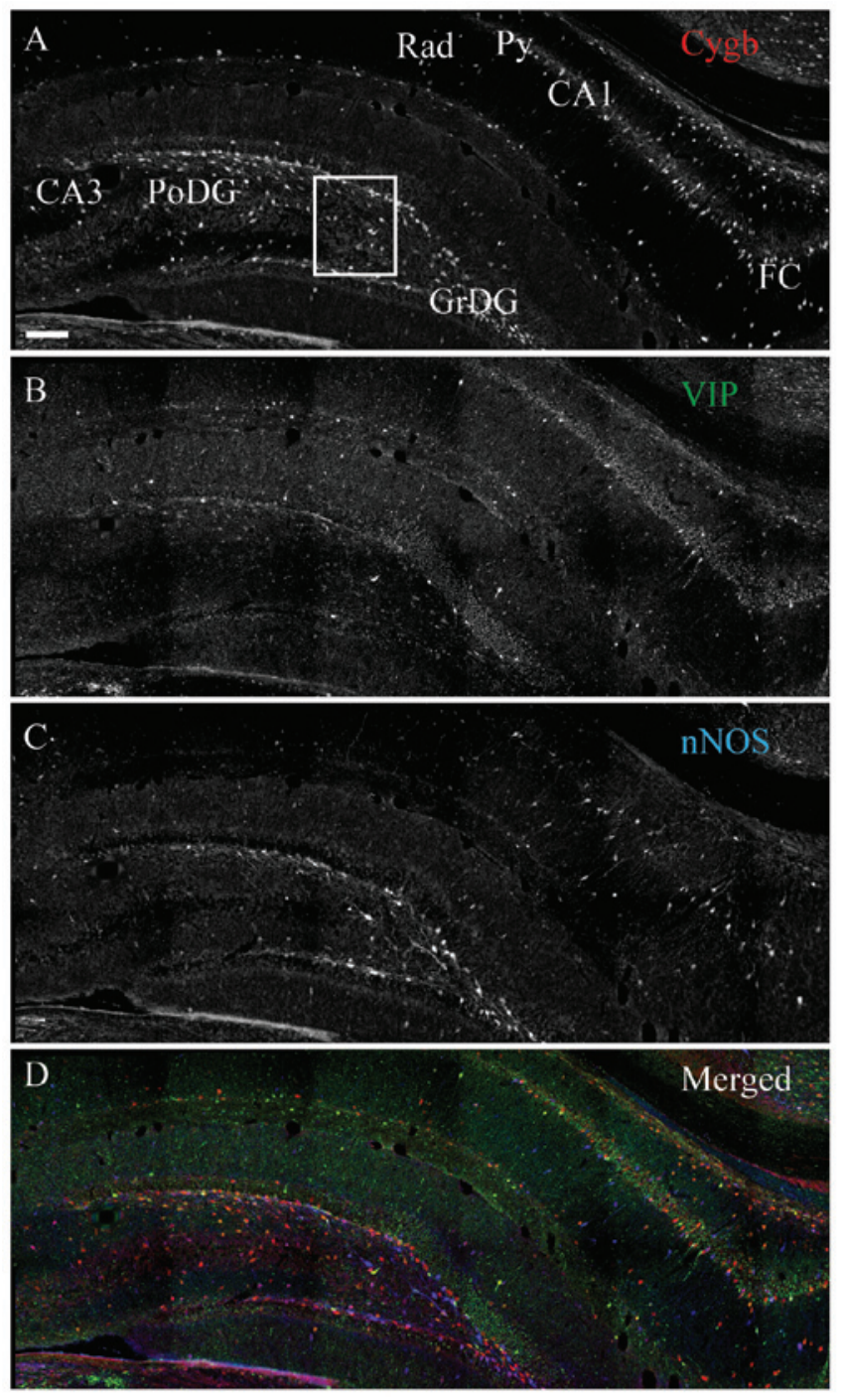

Figure 3. Cytoglobin (Cygb), vasoactive intestinal peptide (VIP) and neuronal nitric oxide synthase (nNOS) expression in the rat hippocampus. Immunohistochemical staining of (A) Cygb, (B) VIP and (C) nNOS. (D) The merged image of A-C is shown. VIP-immunoreactivity (ir) (green) neurons were found to co-localize with the Cygb-ir (red) and nNOS-ir (cyan) neurons. The area within the white box is magnified in Fig. 6. CA1-3, corun ammonis 1-3; FC, fasio larumcinereum; GrDG, striatum granulosum of dentate gyrus; PoDG, polymorph layer of DG; Py, pyramidal cell layer; Rad, stratum radiatum. (A-D) Scale bar, $200 \mu \mathrm{m}$.

\section{Discussion}

Despite intensive research (32) over the past 12 years, the function of Cygb remains an enigma. To the best of our knowledge, the present study is the first detailed characterization of the neurochemical phenotype of Cygb-ir neurons in the rat hippocampus and can, in combination with other methods, contribute to an improved understanding of the Cygb function. The study was performed in the rat model as the employed antibodies produced an optimal immunohistochemical staining, as opposed to the mouse.

Clarifying the function of a protein in the brain is challenging due to the high complexity and the interconnections of the brain structures. Therefore, having tools that minimize the complexity and provide a clearer functional readout are highly advantageous. We have previously used neurochemical phenotyping to obtain 

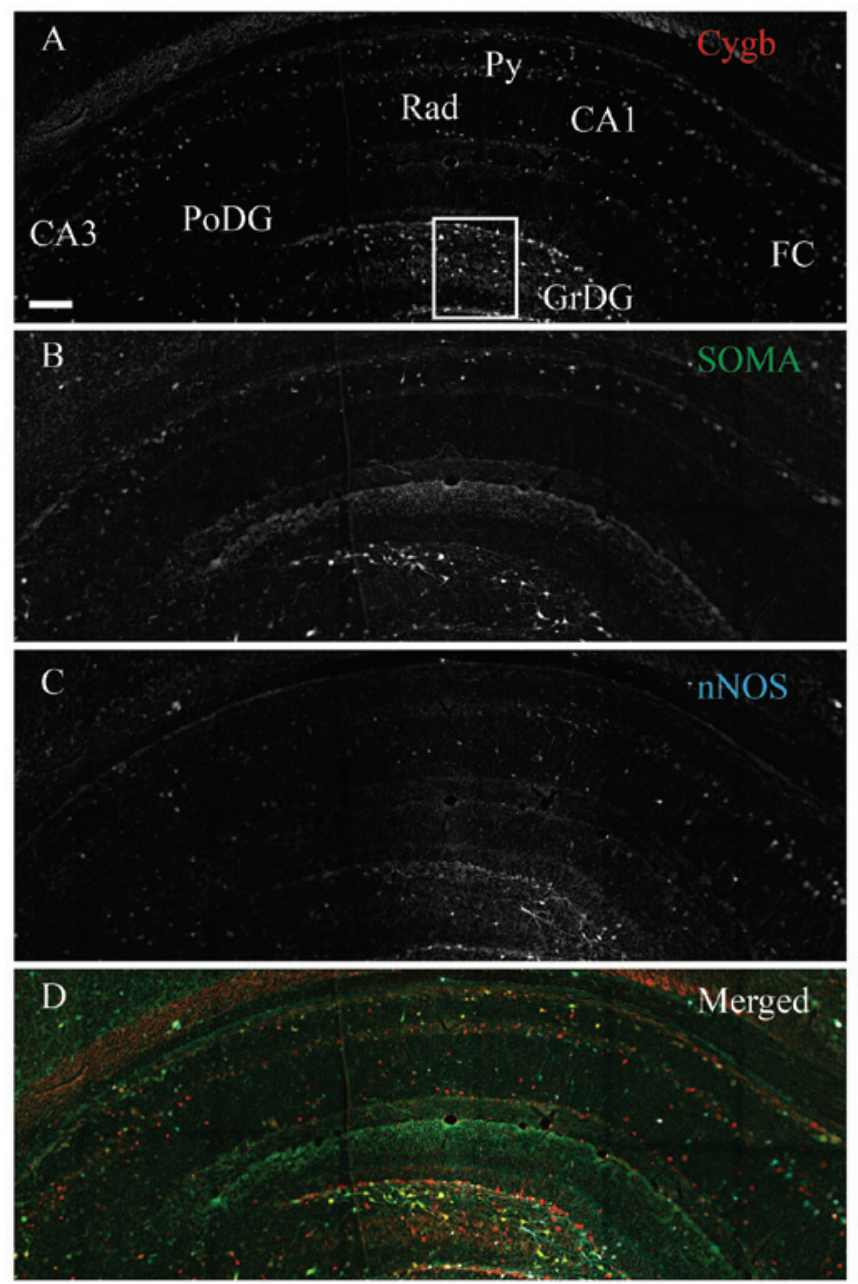

Figure 4. Cytoglobin (Cygb), somastostain (SOMA) and neuronal nitric oxide synthase (nNOS) expression in the rat hippocampus. Immunohistochemical staining of (A) Cygb, (B) SOMA and (C) nNOS. (D) The merged image of A-C is shown. As for vasoactive intestinal peptide, SOMA-immunoreactivity (ir) (green) neurons primarily belonged to the sub-population of Cygb-ir (red) neurons, also co-localizing nNOS-ir (cyan). The area within the white box is magnified in Fig. 6. CA1-3, corun ammonis 1-3; FC, fasio larumcinereum; GrDG, striatum granulosum of dentate gyrus; PoDG, polymorph layer of DG; Py, pyramidal cell layer; Rad, stratum radiatum. Scale bar, $200 \mu \mathrm{m}$.

surrogate markers of the protein of interest in knock-out mice in order to study the fate of neurons that would have expressed the protein of interest. This enabled the study of whether these neurons were more prone to hypoxia-induced immediate early gene expression and cell death (33).

The principal novel finding of the present study is the high degree of co-localization between Cygb and the two markers, PV and HO-1, in the hippocampus of which only a subset of neurons also co-express nNOS-ir. The high degree of Cygb-ir/PV-ir co-expression therefore shows for the first time that $\mathrm{Cygb}$-expressing neurons are primarily inhibitory interneurons. The high degree of co-expression with HO-1 is notable as HO-1 is an oxidative stress-inducible protein and enzymatic degradation of heme leads to the production of iron, biliverdin (an antioxidant) and CO (34). Cygb may therefore either be a heme substrate for HO-1 or alternatively, Cygb may, in these $\mathrm{HO}-1$-expressing neurons, regulate the level of the gas-neurontransmitter $\mathrm{CO}$ produced by $\mathrm{HO}-1$ in a similar way to that proposed for NO $(18,19,21)$.
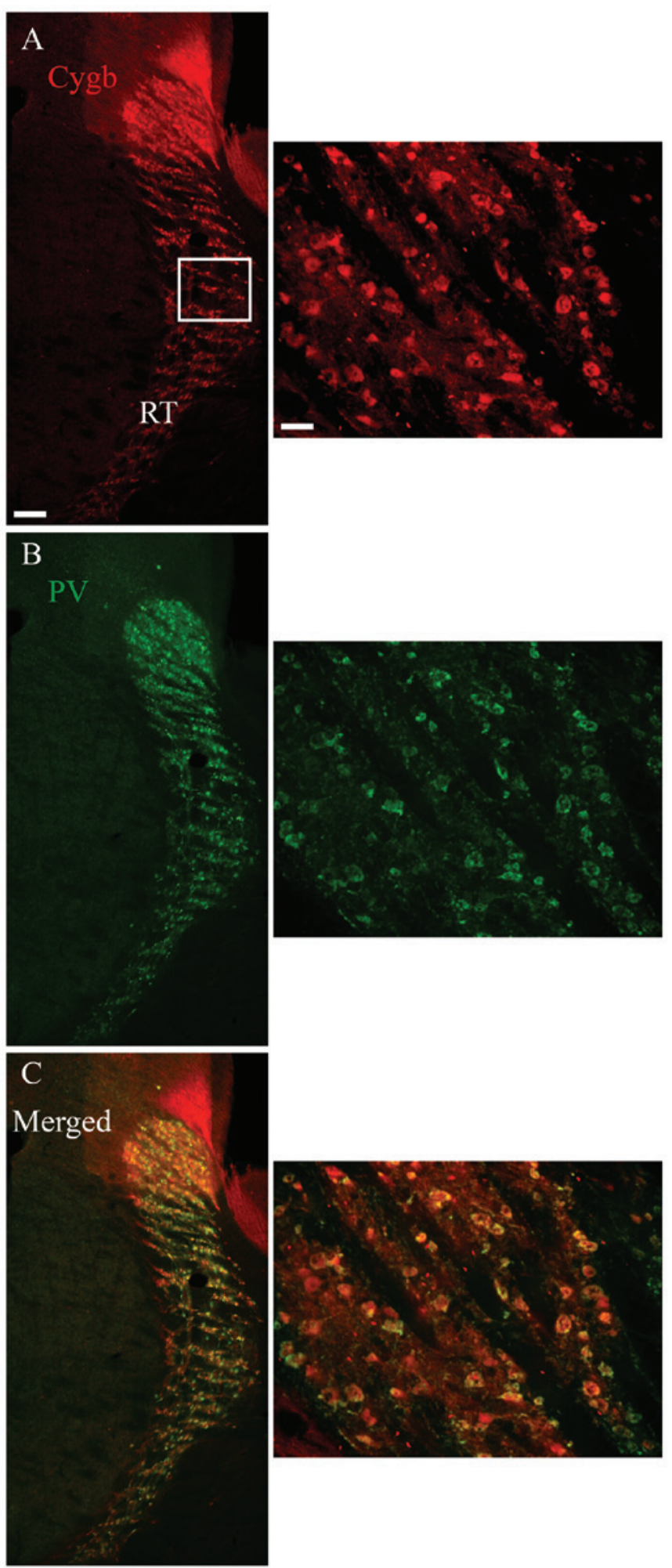

Figure 5. Co-localization of cytoglobin (Cygb)-immunoreactivity (ir) and parvalbumin (PV)-ir in the reticular thalamic nucleus. (A) Intense Cygb-ir (red) and (B) PV-ir (green) were observed in the reticular thalamic nucleus (RT) of the rat brain. (C) All the PV-ir neurons co-expressed with Cygb-ir. The images on the right side are higher magnifications of the area within the white square in (A). Scale bar, (A-C) $200 \mu \mathrm{m}$ and $25 \mu \mathrm{m}$ in high magnifications.

Notably, in association with the putative role of $\mathrm{Cygb}$ in neuroprotection, a selective reduction of PV-ir neurons were observed in the hippocampus of the AD patients and transgenic animal models of AD (35-38). Of note, HO-1-ir 

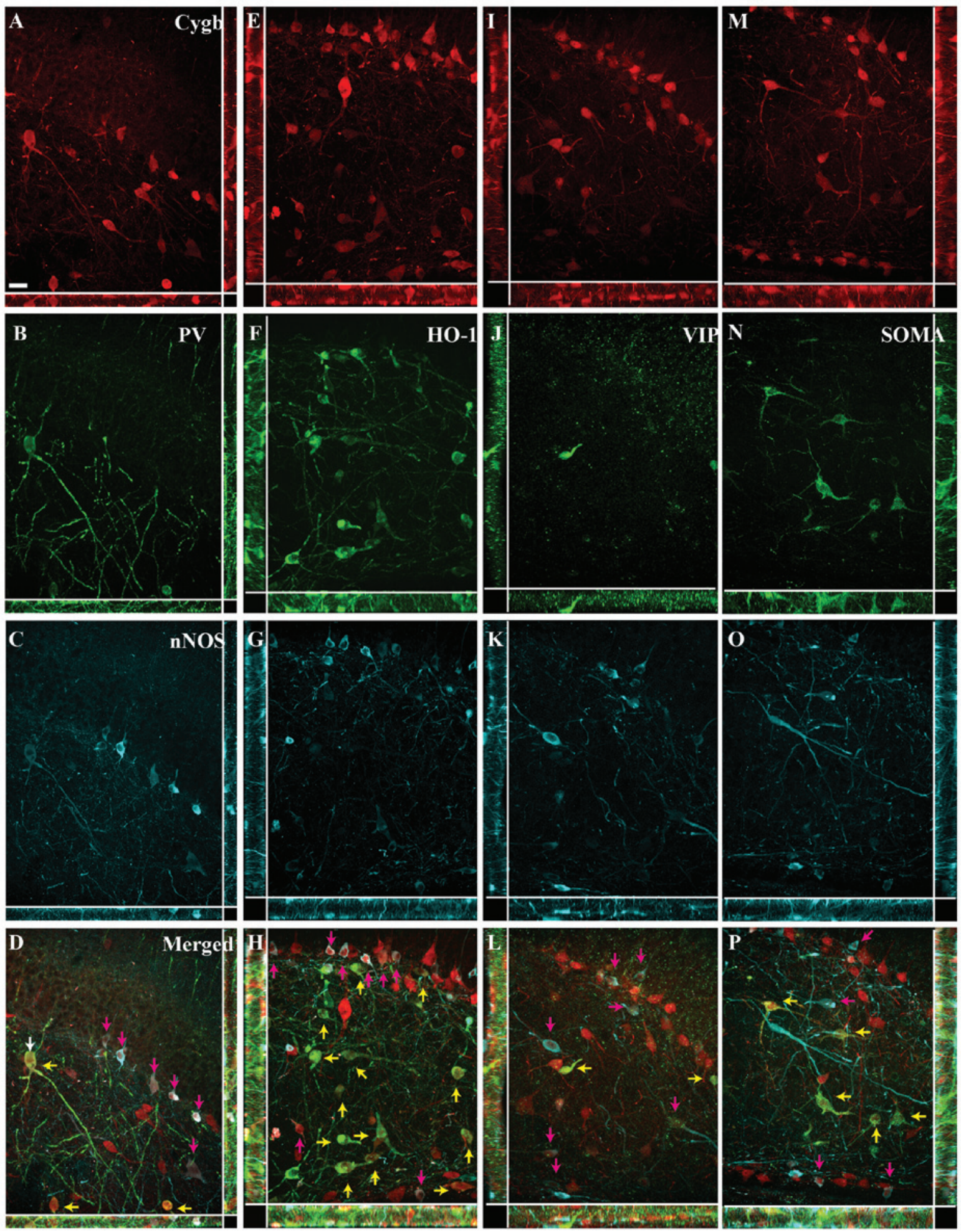

Figure 6. High magnification images of the white squares in Figs. 1D-4D. (A-D) A Z-stack image of dentate gyrus neurons expressing cytoglobin (Cygb) (red), parvalbumin (PV) (green) and neuronal nitric oxide synthase (nNOS) (cyan). The yellow arrows (D) indicate that certain cells co-store Cygb and PV, the purple arrows indicate the cells co-storing Cygb and nNOS and the white arrow show a cell co-expressing all three. Similarly, (E-H) a Z-stack image of co-expression with heme-oxygenase-1 (HO-1) (green). The yellow arrows (H) indicate that certain cells co-store Cygb and HO-1 and the purple arrows indicate the cells co-storing Cygb and nNOS. (I-L) Co-localization with vasoactive intestinal peptide (VIP) (green) and (M-P) with somastostain (SOMA) is shown. The white vertical and horizontal lines indicate the $\mathrm{Y}-\mathrm{Z}$ and $\mathrm{X}-\mathrm{Z}$ axis, respectively. Scale bar, $25 \mu \mathrm{m}$.

has been shown to be upregulated in hippocampal neurons of the AD patients (39) and to contribute to the pathology by excessive production of free iron (40-42). The high degree of co-expression with Cygb indicates that Cygb could be a heme substrate for HO-1 enzymatic activity and thereby a source for excessive iron production, which contributes to the pathology of AD. Taken together, this indirectly indicates that expressing Cygb alone does not provide neurons with a selective 
protection against neurodegenerative cell death, which is in line with the lack of selective sparring of Cygb-expressing neurons following brain ischemia (22).

Cygb-ir was also co-expressed in SOMA-ir and VIP-ir neurons to a high degree. These neurons, however, belonged primarily to the neurons that also expressed nNOS-ir. VIP and SOMA have a broad range of functions in the central nervous system, however, in association with neuronal protection, a reduced number of viable SOMA neurons were found in human AD brains $(43,44)$, whereas VIP have been shown to exert a level of protection in neurodegenerative disorders (45-47). These observations indirectly show that despite SOMA, neurons also express Cygb and they are still not spared in neurodegenerative disorders, thus questioning if $\mathrm{Cygb}$ at endogenous levels functions in neuronal protection.

Future studies using Cygb null mice $(20,48)$ would be highly beneficially for studying the fate/function of neurons expressing PV, HO-1, SOMA and VIP. These studies will show whether a lack of Cygb affects neuronal survival or normal cell physiology by using these four proteins as markers. Based on the large expression of Cygb in the hippocampus, it is highly likely that Cygb has an important function in hippocampal normal physiology. The results in the present study will be a significant aid for future studies in elucidating any functional roles.

\section{Acknowledgements}

The authors are most grateful to Professor Eero Vasar and the Centre of Excellence for Translational Medicine for providing excellent working facilities and to Dr Brent M. Witgen for the helpful discussion of the manuscript. This work was supported by the Estonia Research Council (PUT120) and the European Regional Development Fund.

\section{References}

1. McEwen BS: Physiology and neurobiology of stress and adaptation: central role of the brain. Physiol Rev 87: 873-904, 2007.

2. Hampel H, Bürger K, Teipel SJ, Bokde AL, Zetterberg H and Blennow K: Core candidate neurochemical and imaging biomarkers of Alzheimer's disease. Alzheimers Dement 4 38-48, 2008.

3. Harrison PJ: The hippocampus in schizophrenia: a review of the neuropathological evidence and its pathophysiological implications. Psychopharmacology (Berl) 174: 151-162, 2004.

4. Michaelis EK: The Clinical Neurobiology Of The Hippocampus: An Integrative View. Bartsch T (ed). Oxford University Press, Oxford, pp. 59-70, 2012.

5. Burmester T, Ebner B, Weich B and Hankeln T: Cytoglobin: a novel globin type ubiquitously expressed in vertebrate tissues. Mol Biol Evol 19: 416-421, 2002.

6. Trent JT III and Hargrove MS: A ubiquitously expressed human hexacoordinate hemoglobin. J Biol Chem 277: 19538-19545, 2002.

7. Fago A, Hundahl C, Malte H and Weber RE: Functional properties of neuroglobin and cytoglobin. Insights into the ancestral physiological roles of globins. IUBMB Life 56: 689-696, 2004.

8. Schmidt M, Gerlach F, Avivi A, et al: Cytoglobin is a respiratory protein in connective tissue and neurons, which is up-regulated by hypoxia. J Biol Chem 279: 8063-8069, 2004.

9. Hundahl CA, Allen GC, Hannibal J, et al: Anatomical characterization of cytoglobin and neuroglobin mRNA and protein expression in the mouse brain. Brain Res 1331: 58-73, 2010.

10. Hundahl CA, Hannibal J, Fahrenkrug J, Dewilde S and Hay-Schmidt A: Neuroglobin expression in the rat suprachiasmatic nucleus: colocalization, innervation, and response to light. J Comp Neurol 518: 1556-1569, 2010.
11. Mammen PP, Shelton JM, Ye Q, et al: Cytoglobin is a stress-responsive hemoprotein expressed in the developing and adult brain. J Histochem Cytochem 54: 1349-1361, 2006.

12. Hundahl CA, Kelsen J and Hay-Schmidt A: Neuroglobin and cytoglobin expression in the human brain. Brain Struct Funct 218: 603-609, 2013

13. Hundahl CA, Elfving B, Müller HK, Hay-Schmidt A and Wegener G: A gene-environment study of cytoglobin in the human and rat hippocampus. PLoS One 8: e63288, 2013.

14. Fordel E, Thijs L, Martinet W, Schrijvers D, Moens L and Dewilde S: Anoxia or oxygen and glucose deprivation in SH-SY5Y cells: a step closer to the unraveling of neuroglobin and cytoglobin functions. Gene 398: 114-122, 2007.

15. Fordel E, Thijs L, Moens L and Dewilde S: Neuroglobin and cytoglobin expression in mice. Evidence for a correlation with reactive oxygen species scavenging. FEBS J 274: 1312-1317, 2007.

16. Li D, Chen XQ, Li WJ, Yang YH, Wang JZ and Yu AC: Cytoglobin up-regulated by hydrogen peroxide plays a protective role in oxidative stress. Neurochem Res 32: 1375-1380, 2007.

17. Hodges NJ, Innocent N, Dhanda S and Graham M: Cellular protection from oxidative DNA damage by over-expression of the novel globin cytoglobin in vitro. Mutagenesis 23: 293-298, 2008.

18. Gardner AM, Cook MR and Gardner PR: Nitric-oxide dioxygenase function of human cytoglobin with cellular reductants and in rat hepatocytes. J Biol Chem 285: 23850-23857, 2010.

19. Halligan KE, Jourd'heuil FL and Jourd'heuil D: Cytoglobin is expressed in the vasculature and regulates cell respiration and proliferation via nitric oxide dioxygenation. J Biol Chem 284: 8539-8547, 2009.

20. Singh S, Canseco DC, Manda SM, et al: Cytoglobin modulates myogenic progenitor cell viability and muscle regeneration. Proc Natl Acad Sci USA 111: E129-E138, 2014.

21. Tian SF, Yang HH, Xiao DP, et al: Mechanisms of neuroprotection from hypoxia-ischemia (HI) brain injury by up-regulation of cytoglobin (CYGB) in a neonatal rat model. J Biol Chem 288: 15988-16003, 2013.

22. Raida Z, Reimets R, Hay-Schmidt A and Hundahl CA: Effect of permanent middle cerebral artery occlusion on Cytoglobin expression in the mouse brain. Biochem Biophys Res Commun 424: 274-278, 2012

23. Hundahl CA, Fahrenkrug J, Hay-Schmidt A, Georg B, Faltoft B and Hannibal J: Circadian behaviour in neuroglobin deficient mice. PLoS One 7: e34462, 2012.

24. Hao MM, Bornstein JC and Young HM: Development of myenteric cholinergic neurons in ChAT-Cre;R26R-YFP mice. J Comp Neurol 521: 3358-3370, 2013.

25. Yan $\mathrm{H}$ and Keast JR: Neurturin regulates postnatal differentiation of parasympathetic pelvic ganglion neurons, initial axonal projections, and maintenance of terminal fields in male urogenital organs. J Comp Neurol 507: 1169-1183, 2008.

26. Cox DJ and Racca C: Differential dendritic targeting of AMPA receptor subunit mRNAs in adult rat hippocampal principal neurons and interneurons. J Comp Neurol 521: 1954-2007, 2013.

27. Spiegel AM, Koh MT, Vogt NM, Rapp PR and Gallagher M: Hilar interneuron vulnerability distinguishes aged rats with memory impairment. J Comp Neurol 521: 3508-3523, 2013.

28. Fahrenkrug J, Buhl T and Hannibal J: PreproPACAP-derived peptides occur in VIP-producing tumours and co-exist with VIP. Regul Pept 58: 89-98, 1995.

29. SchwallerB,DickJ,DhootG, et al:Prolonged contraction-relaxation cycle of fast-twitch muscles in parvalbumin knockout mice. Am J Physiol 276: C395-C403, 1999.

30. Stephenson-Jones M, Ericsson J, Robertson B and Grillner S: Evolution of the basal ganglia: dual-output pathways conserved throughout vertebrate phylogeny. J Comp Neurol 520: 2957-2973, 2012.

31. Hundahl CA, Kelsen J, Dewilde S and Hay-Schmidt A: Neuroglobin in the rat brain (II): co-localisation with neurotransmitters. Neuroendocrinology 88: 183-198, 2008.

32. Burmester T and Hankeln T: Function and evolution of vertebrate globins. Acta Physiol (Oxf). May 8, 2014 (Epub ahead of print).

33. Hundahl CA, Luuk H, Ilmjärv S, et al: Neuroglobin-deficiency exacerbates Hif1A and c-FOS response, but does not affect neuronal survival during severe hypoxia in vivo. PLoS One 6: e28160, 2011.

34. Schipper HM: Heme oxygenase-1: transducer of pathological brain iron sequestration under oxidative stress. Ann NY Acad Sci 1012: 84-93, 2004.

35. Verret L, Mann EO, Hang GB, et al: Inhibitory interneuron deficit links altered network activity and cognitive dysfunction in Alzheimer model. Cell 149: 708-721, 2012. 
36. Takahashi H, Brasnjevic I, Rutten BP, et al: Hippocampa interneuron loss in an APP/PS1 double mutant mouse and in Alzheimer's disease. Brain Struct Funct 214: 145-160, 2010.

37. Brady DR and Mufson EJ: Parvalbumin-immunoreactive neurons in the hippocampal formation of Alzheimer's diseased brain. Neuroscience 80: 1113-1125, 1997.

38. Popović M, Caballero-Bleda M, Kadish I and Van Groen T: Subfield and layer-specific depletion in calbindin-D28K, calretinin and parvalbumin immunoreactivity in the dentate gyrus of amyloid precursor protein/presenilin 1 transgenic mice. Neuroscience 155: 182-191, 2008.

39. Schipper HM, Cissé S and Stopa EG: Expression of heme oxygenase-1 in the senescent and Alzheimer-diseased brain. Ann Neurol 37: 758-768, 1995.

40. Schipper HM: Heme oxygenase-1: role in brain aging and neurodegeneration. Exp Gerontol 35: 821-830, 2000.

41. Schipper HM: Glial HO-1 expression, iron deposition and oxidative stress in neurodegenerative diseases. Neurotox Res 1: 57-70, 1999.

42. Beal MF: Metabolic disorders and neurotoxicology. Curr Opin Neurol 8: 467-468, 1995.

43. Dournaud P, Cervera-Pierot P, Hirsch E, et al: Somatostatin messenger RNA-containing neurons in Alzheimer's disease: an in situ hybridization study in hippocampus, parahippocampal cortex and frontal cortex. Neuroscience 61: 755-764, 1994.
44. Rossor MN, Emson PC, Mountjoy CQ, Roth M and Iversen LL: Reduced amounts of immunoreactive somatostatin in the temporal cortex in senile dementia of Alzheimer type. Neurosci Lett 20: 373-377, 1980.

45. Delgado M, Varela N and Gonzalez-Rey E: Vasoactive intestinal peptide protects against beta-amyloid-induced neurodegeneration by inhibiting microglia activation at multiple levels. Glia 56: 1091-1103, 2008

46. Delgado M and Ganea D: Neuroprotective effect of vasoactive intestinal peptide (VIP) in a mouse model of Parkinson's disease by blocking microglial activation. FASEB J 17: 944-946, 2003.

47. Offen D, Sherki Y, Melamed E, Fridkin M, Brenneman DE and Gozes I: Vasoactive intestinal peptide (VIP) prevents neurotoxicity in neuronal cultures: relevance to neuroprotection in Parkinson's disease. Brain Res 854: 257-262, 2000.

48. Thuy le TT, Morita T, Yoshida K, et al: Promotion of liver and lung tumorigenesis in DEN-treated cytoglobin-deficient mice. Am J Pathol 179: 1050-1060, 2011 